\title{
新型一维有机-无机聚合物: $\left[\left\{\mathrm{Ca}(\mathrm{DMF})_{5}\right\}_{2}-\right.$ $\left.\mathrm{SiMo}_{12} \mathrm{O}_{40}\right]_{n}$ 的合成、结构及热性质 ${ }^{*}$
}

\author{
王敬平 吴强牛景扬
}

(河南大学化学化工学院, 开封 475001)

\begin{abstract}
摘要以硅钼酸、氯化钻和 $\mathrm{N}, \mathrm{N}$-二甲基甲酰胺为原料合成了以聚金属氧酸盐为构筑 块的一维链状有机-无机聚合物 $\left[\left\{\mathrm{Ca}(\mathrm{DMF})_{5}\right\}_{2} \mathrm{SiMo}_{12} \mathrm{O}_{40}\right]_{n}$. 化合物的晶体属于单斜晶系, $\mathrm{P} 2{ }_{1} / \mathrm{n}$ 空间群, $a=1.3379(3), b=1.9796(4), c=1.4574(3) \mathrm{nm}, \beta=92.24(3)^{\circ}, V=3.8568(13)$ $\mathrm{nm}^{3}, Z=2$. 由 7589 个可观测衍射 $[I \geqslant 2 \sigma(I)]$ 用于精修所有的结构参数, 得一致性因子 $R_{1}=0.083, w R_{2}=0.2065$. 结构测定结果表明, 化合物中 $\mathrm{Ca}^{2+}$ 离子为七配位, 五角双锥构 型, 配位氧原子分别来自 6 个 $\mathrm{DMF}$ 分子和 1 个杂多阴离子的端氧原子. 金属离子与杂 多阴离子的端氧成键并通过 Ca-O-Mo 连接与杂多阴离子骨架相连. 化合物中相邻的 $\left\{\mathrm{Ca}(\mathrm{DMF})_{5}\right\}_{2} \mathrm{SiMo}_{12} \mathrm{O}_{40}$ 结构单元通过 $\mathrm{Ca}-\mathrm{O}-\mathrm{Ca}$ 形式桥联形成新奇的一维无限链; 化合 物的 IR 光谱和 X 射线衍射结果表明, 有机给体与杂多阴离子之间在固态条件下有较强 的相互作用; UV 光谱表明在稀的乙腈和水的混合溶液中, 杂多阴离子与外界阳离子在 溶剂作用下处于解离状态; TG/DTA 曲线表明，化合物的失重过程分三步进行，化合物 中杂多阴离子的热稳定性与硅钼酸中 $\mathrm{SiMo}_{12} \mathrm{O}_{40}{ }^{4-}$ 的相比有明显升高.
\end{abstract}

\section{关键词合成 晶体结构 热性质}

聚金属氧酸盐以其优越的理化性质，已经成为构造新型分子基功能材料的重要无机构筑 块 ${ }^{[1]}$. 作为电子受体的聚金属氧酸盐可以与有机 $\pi$ 电子给体如 TTF, ET 和有机金属茂合物结 合, 形成有机-无机复合物, 在光, 电, 磁和超导领域有潜在的应用前景 ${ }^{[2 \sim 4]}$. 在这些化合物的 结构中, 有机给体与杂多阴离子之间虽然存在弱相互作用, 但是没有直接的联系. 通常, 这类 化合物晶体的稳定性较差. 根据分子设计的原理, 我们向有机-无机复合物的结构中引人金属 离子, 目的是使金属离子与有机给体配位, 以期增强他们晶体的稳定性, 并希望产生新的结构 和性质. 有文献报道过金属阳离子与同多聚金属氧酸盐中的 $\mathrm{MoO}_{6}$ 八面体的端氧配位 ${ }^{[5,6]}$, 但 金属离子与 Keggin 结构杂多阴离子的端氧配位尚未见文献报道. 这里, 我们报道一个新奇的 以杂多聚金属氧酸盐为构筑块的有机-无机聚合物 $\left[\left\{\mathrm{Ca}(\mathrm{DMF})_{5}\right\}_{2} \mathrm{SiMo}_{12} \mathrm{O}_{40}\right]_{n}$ 的合成及表征. 结 构测定结果表明，在标题化合物的结构中，金属阳离子与杂多阴离子的端氧配位，毗邻的 $\left[\mathrm{Ca}(\mathrm{DMF})_{5}\right]_{2} \mathrm{SiMo}_{12} \mathrm{O}_{40}$ 结构单元利用相邻的金属离子通过与有机给体配位相互连接, 形成一 维链状聚合结构. 


\section{1 实验与结构分析}

\section{1 化合物的合成}

$\mathrm{H}_{4} \mathrm{SiMo}_{12} \mathrm{O}_{40} \cdot n \mathrm{H}_{2} \mathrm{O}$ 参照文献[7]方法合成, 并经红外光谱表征为目标化合物.

将(0.24 g, $2.2 \mathrm{mmol}) \mathrm{CaCl}_{2}$ 加人 $15 \mathrm{~mL} \mathrm{H}_{4} \mathrm{SiMo}_{12} \mathrm{O}_{4}(2.0 \mathrm{~g}, 2.2 \mathrm{mmol})$ 的水溶液中, 在 $90^{\circ} \mathrm{C}$ 下搅拌反应, 直到溶液蒸发近干, 其间有刺激性气体放出. 然后停止反应, 将析出的固体干燥 后放置待用.

称取 $2.0 \mathrm{~g}$ 上述干燥样品溶于 $15 \mathrm{~mL}$ 乙腈和水的混合溶剂中(体积比约 5:2), 向溶液中缓 慢滴加 $1 \mathrm{~mL} \mathrm{DMF}$ 溶液, 在 $70^{\circ} \mathrm{C}$ 下反应 $10 \mathrm{~min}$ 后冷却, 过滤, 向滤液中加人少量混合溶剂后放 置在暗处缓慢挥发. 数日后, 有黄色晶体析出. 按实验式 $\mathrm{C}_{30} \mathrm{H}_{70} \mathrm{Ca}_{2} \mathrm{Mo}_{12} \mathrm{~N}_{10} \mathrm{O}_{54} \mathrm{Si}$, 化合物的元 素分析结果为(\%): 计算值: C, 13.36; H, 2.59; N, 5.19. 实验值: C, 13.24; H, 2.26; N, 5.32.

\section{2 化合物的结构分析}

选取 $0.32 \mathrm{~mm} \times 0.26 \mathrm{~mm} \times 0.22 \mathrm{~mm}$ 的黄色单晶样品, 在 Rigaku-RAXIS-IV 面探仪上, 采用 $\mathrm{MoK} \alpha$ 射线, 各衍射数据在 $293(2) \mathrm{K}$ 下收集, 可观察衍射数据 7589 个. 结构分析表明, 该晶体 属单斜晶系, $\mathrm{P} 2{ }_{1} / \mathrm{n}$ 空间群. 晶胞参数: $a=1.3379(3), b=1.9796(4), c=1.4574(3) \mathrm{nm}, \beta=$ $92.24(3)^{\circ}, V=3.8568(13) \mathrm{nm}^{3}, Z=2, D c=2.320 \mathrm{~g} \cdot \mathrm{cm}^{-3}, F(000)=2620$. 所有数据经 $L P$ 因子校 正. 用直接法得到全部非氢原子坐标, 并经全矩阵最小二乘法精修, 最终偏离因子 $R_{1}=0.083$, $w R_{2}=0.2065, G O F=1.105, \Delta \rho_{\max }=0.1689, \Delta \rho_{\min }=-0.1330\left(\mathrm{e} \cdot \mathrm{nm}^{-3}\right)$, 所有计算均使用 SHLEXS-97 程序, 在 P III-773 计算机上进行.

\section{3 化合物的表征}

( i ) IR 光谱. 采用溴化钾压片, 用 Nicolet170 Fourier红外光谱仪测定化合物的红外光谱, 主要数据如下: $1654\left(v_{\mathrm{C}=\mathrm{O}}\right), 944\left(v_{\mathrm{Mo}-\mathrm{O}_{\mathrm{d}}}\right), 898\left(v_{\mathrm{Si}_{-} \mathrm{a}}\right), 866\left(v_{\mathrm{Mo}-\mathrm{O}_{\mathrm{b}}}\right), 797\left(v_{\mathrm{Mo}-\mathrm{O}_{\mathrm{c}}}\right)$.

(ii) UV 光谱. 用岛津 UV-250 光谱仪在 5:2 乙腈和水的混合溶剂中测定了化合物的紫 外光谱, 测定范围 $200 \sim 500 \mathrm{~nm}$, 在 $\lambda=235 \mathrm{~nm}$ 处有明显吸收, 此峰归属于杂多阴离子中 $\mathrm{O} \rightarrow \mathrm{Mo}$ 的荷迁移峰.

(iii) 差热及热重曲线. 由 Perkin Elmer-7 差热热重分析仪给出(空气气氛, 升温速度 $10^{\circ} \mathrm{C}$ /min, 测定范围从室温至 $600^{\circ} \mathrm{C}$ ).

\section{2 结果与讨论}

\section{1 合成}

影响标题化合物合成的因素主要有溶剂及反应温度. 选择合适的混合溶剂对晶体生长及 所获晶体质量的好坏极为重要. 将标题化合物溶解在乙腈或水的溶液中, 都得不到合适的晶 体. 反应时加热温度过高, 杂多酸盐易分解, 得不到所要的产物, 所以加热温度应严格控制. 此外，应该避免在光照条件下培养晶体，否则，所获晶体易变色. 这是因为在光照条件下，有 机给体与杂多阴离子之间易发生分子内电荷转移, 使杂多阴离子中的 $\mathrm{Mo}^{6+}$ 离子还原.

\subsection{IR 光谱}

红外光谱结果表明标题化合物仍保持基本 Keggin 结构特征峰. 比较标题化合物和 $\mathrm{H}_{4} \mathrm{SiMo}_{12} \mathrm{O}_{40}$ 的红外光谱 ${ }^{[7]}$, 标题化合物的 $\mathrm{Mo}-\mathrm{O}_{\mathrm{d}}$ 振动峰发生了红移, 从 $957 \mathrm{~cm}^{-1}$ 红移至 944 
$\mathrm{cm}^{-1} ; \mathrm{Mo}-\mathrm{O}_{\mathrm{b}}$ 和 $\mathrm{Mo}-\mathrm{O}_{\mathrm{c}}$ 振动峰发生了蓝移, 分别从 $855,770 \mathrm{~cm}^{-1}$ 蓝移至 $866,797 \mathrm{~cm}^{-1} ; \mathrm{Si}^{-} \mathrm{O}_{\mathrm{a}}$ 振 动峰位置基本未变. 以上结果表明阳离子对阴离子有较强的作用. 与此同时, 配体 DMF 的 $\mathrm{C}=\mathrm{O}$ 振动峰由游离的 $\mathrm{DMF}$ 的 $1680 \mathrm{~cm}^{-1}$ 红移至 $1654 \mathrm{~cm}^{-1}$, 这表明 $\mathrm{DMF}$ 是以 $\mathrm{C}=\mathrm{O}$ 键的氧原子与 金属离子发生配位作用, 晶体结构数据给出了同样的证明. 这是由于 DMF上的氧原子与金属离子 配位, 氧上电子向金属离子转移, 导致氧上电子云密度降低. 因此, $\mathrm{C}=\mathrm{O}$ 键的伸缩振动频率降低.

\section{$2.3 \mathrm{UV}$ 光谱}

$\left[\left\{\mathrm{Ca}(\mathrm{DMF})_{5}\right\}_{2} \mathrm{SiMo}_{12} \mathrm{O}_{40}\right]_{n}$ 在乙腈和水的混合溶剂中的紫外光谱与同样条件下 $\mathrm{H}_{4} \mathrm{SiMo}_{12} \mathrm{O}_{40}$ 的紫外光谱相比较, 无明显差别, 都在 $\lambda=235 \mathrm{~nm}$ 处有一吸收峰, 此峰归属于杂 多阴离子中 $\mathrm{O} \rightarrow \mathrm{Mo}$ 的荷移跃迁, 与文献报道过的现象类似 ${ }^{[8]}$. 紫外光谱结果表明, 标题化合 物中的杂多阴离子和金属离子在稀溶液中处于解离状态, 由于受溶剂效应的影响, 彼此之间 无相互作用.

\section{4 晶体结构}

非氢原子坐标和等效各向同性参数、主要键长和键角在表 1 3 中给出.

表 1 原子坐标 $\left(\times 10^{4}\right)$ 和各向同性的热参数 $\left(\times 10^{5} \mathrm{~nm}^{2}\right)$

\begin{tabular}{|c|c|c|c|c|}
\hline & $x$ & $y$ & $z$ & $U(\mathrm{eq})^{\mathrm{a})}$ \\
\hline Si1 & 0 & 0 & 5000 & $30(1)$ \\
\hline Mo1 & $-2127(1)$ & $-463(1)$ & $6239(1)$ & $43(1)$ \\
\hline Mo2 & $2085(1)$ & $-1098(1)$ & 4951(1) & $41(1)$ \\
\hline Mo3 & $-1372(1)$ & $-1462(1)$ & $4365(1)$ & $54(1)$ \\
\hline Mo4 & $537(1)$ & $-863(1)$ & 2951(1) & $48(1)$ \\
\hline Mo5 & $95(1)$ & $-1446(1)$ & $6442(1)$ & $48(1)$ \\
\hline Mo6 & $-1715(1)$ & $60(1)$ & $3103(1)$ & $51(1)$ \\
\hline $\mathrm{Ca} 1$ & $-3753(2)$ & $138(1)$ & $842(2)$ & $39(1)$ \\
\hline $\mathrm{O} 1$ & $-3065(7)$ & $-681(7)$ & $6879(7)$ & $83(4)$ \\
\hline $\mathrm{O} 2$ & $3093(7)$ & $-1578(5)$ & $4952(8)$ & $73(3)$ \\
\hline $\mathrm{O} 3$ & $-2109(7)$ & $-2107(5)$ & $4065(7)$ & $61(2)$ \\
\hline O4 & $792(9)$ & $-1319(5)$ & $2045(7)$ & $73(3)$ \\
\hline O5 & $158(9)$ & $-2146(5)$ & $7039(7)$ & $71(3)$ \\
\hline O6 & $-2433(7)$ & $117(5)$ & $2141(6)$ & $63(3)$ \\
\hline $\mathrm{O} 7$ & $-534(8)$ & $-377(7)$ & 2541(13) & $124(6)$ \\
\hline O8 & $1469(9)$ & $-1306(5)$ & $3879(8)$ & $82(3)$ \\
\hline O9 & $-1421(9)$ & $172(5)$ & $7106(8)$ & $85(4)$ \\
\hline O10 & $-2612(10)$ & 271(5) & $5647(7)$ & $85(4)$ \\
\hline O11 & $1268(9)$ & $-1619(7)$ & $5653(8)$ & $96(4)$ \\
\hline $\mathrm{O} 12$ & $-2392(10)$ & $559(7)$ & $3908(8)$ & $100(5)$ \\
\hline O13 & $-387(8)$ & $-1509(7)$ & $3550(12)$ & $118(6)$ \\
\hline $\mathrm{O} 14$ & $-1078(10)$ & $-1080(7)$ & $6771(10)$ & $115(6)$ \\
\hline O15 & $855(10)$ & $-866(7)$ & 7113(8) & 101(5) \\
\hline O16 & $-2038(8)$ & $-748(7)$ & $3584(12)$ & 119(6) \\
\hline $\mathrm{O} 17$ & $-2265(9)$ & $-1028(7)$ & $5273(9)$ & 108(5) \\
\hline $\mathrm{O} 18$ & $-616(9)$ & $-1806(7)$ & $5321(8)$ & 103(5) \\
\hline O19 & $900(10)$ & $-269(6)$ & $4404(8)$ & $88(3)$ \\
\hline $\mathrm{O} 20$ & $-646(9)$ & $621(7)$ & $5435(9)$ & $89(4)$ \\
\hline $\mathrm{O} 21$ & $470(9)$ & $441(7)$ & $5897(9)$ & $95(4)$ \\
\hline $\mathrm{O} 22$ & $-736(10)$ & $497(7)$ & $4423(10)$ & $95(4)$ \\
\hline
\end{tabular}

a) $U(\mathrm{eq})$ 定义为正交 $U_{i j}$ 张量的迹的 $1 / 3$ 
表 2 主要键长 $(\mathrm{nm})$

\begin{tabular}{|c|c|c|c|}
\hline Mo1-O1 & $0.1651(9)$ & Mo1-O10 & $0.1798(11)$ \\
\hline Mo1-O17 & $0.1802(11)$ & Mo1-O14 & $0.1994(10)$ \\
\hline Mo1-O9 & $0.1994(10)$ & Mo1-O20 & $0.2361(12)$ \\
\hline Mo1-O19\#1 & $0.2407(12)$ & $\mathrm{Mo} 2-\mathrm{O} 2$ & $0.1649(9)$ \\
\hline Mo2-O8 & $0.1786(10)$ & Mo2-O11 & $0.1842(10)$ \\
\hline Mo2-O10\#1 & $0.1996(10)$ & Mo2-O12\#1 & $0.2004(10)$ \\
\hline Mo2-O22\#1 & $0.2373(13)$ & Mo2-O19 & $0.2396(14)$ \\
\hline Mo3-O3 & $0.1662(9)$ & Mo3-O13 & $0.1811(12)$ \\
\hline Mo3-O18 & $0.1821(11)$ & Mo3-O16 & $0.2003(11)$ \\
\hline Mo3-O17 & $0.2009(12)$ & Mo3-O21\#1 & $0.2391(14)$ \\
\hline Mo3-O20 & $0.2455(13)$ & Mo4-O4 & $0.1647(10)$ \\
\hline Mo4-O7 & $0.1808(11)$ & Mo4-O9\#1 & $0.1812(10)$ \\
\hline Mo4-O13 & $0.2001(10)$ & Mo4-O8 & $0.2006(10)$ \\
\hline Mo4-O21\#1 & $0.2347(13)$ & Mo4-O19 & $0.2453(12)$ \\
\hline Mo5-O5 & $0.1637(9)$ & Mo5-O15 & $0.1797(10)$ \\
\hline Mo5-O14 & $0.1809(11)$ & Mo5-O18 & $0.1991(9)$ \\
\hline Mo5-O11 & $0.2011(11)$ & Mo5-O20 & $0.2385(13)$ \\
\hline Mo5-O22\#1 & $0.2437(14)$ & Mo6-O6 & $0.1672(9)$ \\
\hline Mo6-O16 & $0.1805(12)$ & Mo6-O12 & $0.1805(11)$ \\
\hline $\mathrm{Mo}(6)-\mathrm{O} 15 \# 1$ & $0.1999(11)$ & Mo6-O7 & $0.2003(12)$ \\
\hline Mo6-O21\#1 & $0.2386(13)$ & Mo6-O22 & $0.2443(14)$ \\
\hline $\mathrm{Ca} 1-\mathrm{O} 5 \mathrm{~A}$ & $0.2279(11)$ & $\mathrm{Ca} 1-\mathrm{O} 3 \mathrm{~A}$ & $0.2292(10)$ \\
\hline $\mathrm{Ca} 1-\mathrm{O} 4 \mathrm{~A}$ & $0.2378(10)$ & $\mathrm{Ca} 1-\mathrm{O} 2 \mathrm{~A}$ & $0.2381(10)$ \\
\hline Ca1-O1A\#2 & $0.2461(8)$ & Ca1-O1A & $0.2470(8)$ \\
\hline Ca1-O6 & $0.2538(9)$ & $\mathrm{Ca} 1-\mathrm{Ca} 1 \# 2$ & $0.4102(5)$ \\
\hline
\end{tabular}

$\# 1-x,-y,-z+1 ; \# 2-x-1,-y,-z$

表 3 主要键角 $\left(^{\circ}\right)$

\begin{tabular}{lr||lr}
\hline O5A-Ca1-O3A & $173.8(5)$ & O5A-Ca1-O4A & $82.2(5)$ \\
O3A-Ca1-O4A & $96.6(4)$ & O5A-Ca1-O2A & $83.5(5)$ \\
O3A-Ca1-O2A & $93.5(4)$ & O4A-Ca1-O2A & $138.9(4)$ \\
O5A-Ca1-O1A\#2 & $92.6(5)$ & O3A-Ca1-O1A\#2 & $93.1(4)$ \\
O4A-Ca1-O1A\#2 & $77.1(3)$ & O2A-Ca1-O1A\#2 & $142.0(3)$ \\
O5A-Ca1-O1A & $94.0(4)$ & O3A-Ca1-O1A & $90.5(4)$ \\
O4A-Ca1-O1A & $144.2(3)$ & O2A-Ca1-O1A & $75.1(3)$ \\
O1A\#2-Ca1-O1A & $67.4(3)$ & O5A-Ca1-O6 & $99.9(5)$ \\
O3A-Ca1-O6 & $74.0(4)$ & O4A-Ca1-O6 & $73.3(3)$ \\
O2A-Ca1-O6 & $71.5(3)$ & O1A\#2-Ca1-O6 & $145.9(3)$ \\
O1A-Ca1-O6 & $141.9(3)$ & Ca1\#2-O1A-Ca1 & $112.6(3)$ \\
\hline
\end{tabular}

$\# 1-x,-y,-z+1 ; \# 2-x-1,-y,-z$

由图 1 所示, 标题化合物是由 2 个与 $\mathrm{DMF}$ 分子配位的金属离子形成的配离子 $\left[\mathrm{Ca}(\mathrm{DMF})_{5}\right]^{2+}$ 和一个硅钼杂多阴离子构成. 2 个晶体学上等价的 $\mathrm{Ca}^{2+}$ 分别对称地分布在杂多阴离子骨架的两 侧, 与杂多阴离子的端氧配位. 化合物中 $\mathrm{Ca}^{2+}$ 离子为七配位, 五角双雉构型(见图 1), 配位氧原 子分别来自 6 个 DMF 分子和 1 个杂多阴离子的端氧原子. 


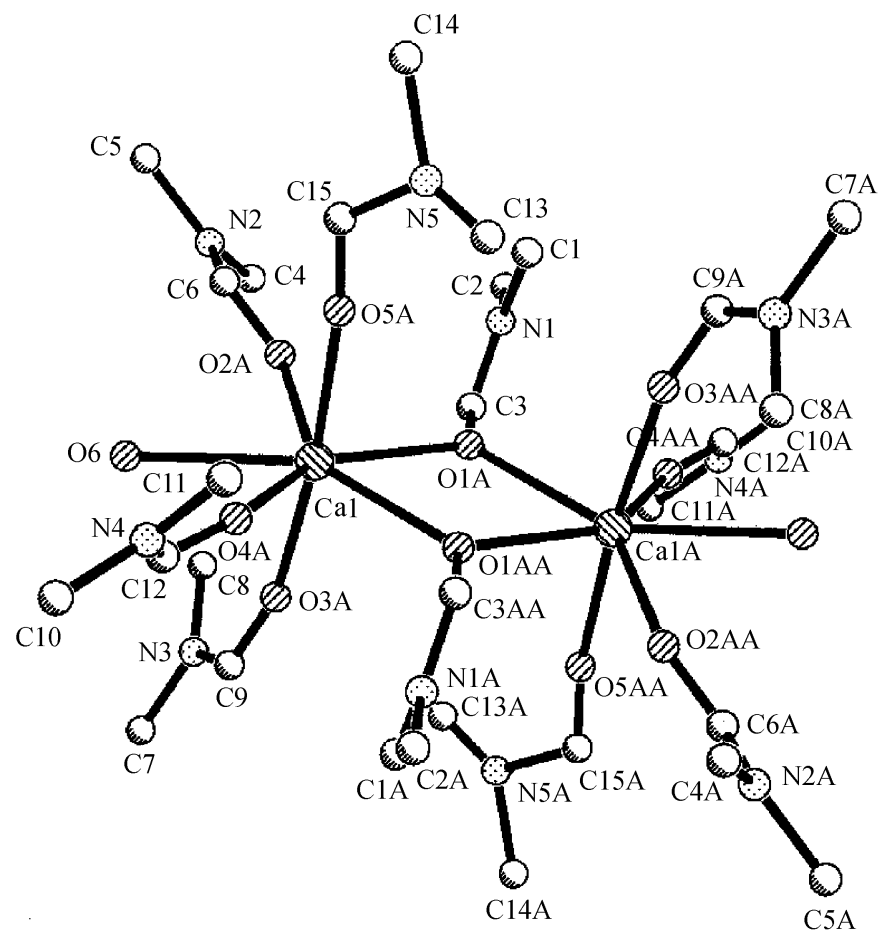

图 $1 \mathrm{Ca}^{2+}$ 的配位环境图

在化合物的结构中, 一方面, 金属离子通过 Ca-O-Mo 连接与杂多阴离子骨架相连; 另一 方面，分属不同 $\left[\mathrm{Ca}(\mathrm{DMF})_{5}\right]_{2} \mathrm{SiMo}_{12} \mathrm{O}_{40}$ 结构单元的相邻的金属离子通过 $\mathrm{DMF}$ 配体相连，以 $\mathrm{Ca}-\mathrm{O}-\mathrm{Ca}$ 形式桥联形成新奇的一维无限链 (见图 2), 其中 O1A 和 O1AA 是 2 个桥氧原子, 分别 属于相邻的不同结构单元的 DMF 配体. Ca-O 键长在 $0.2279(11) \sim 0.2538(9) \mathrm{nm}$ 范围内, 最长的 $\mathrm{Ca}-\mathrm{O}$ 键是连接杂多阴离子端氧的 $\mathrm{Ca}-\mathrm{O} 6$ 键, 键长为 $0.2538(9) \mathrm{nm}$. 以上键长均在文献报道的范 围内 ${ }^{[9]}$. 非键的 $\mathrm{Ca} 1 \cdots \mathrm{Ca} 1 \mathrm{~A}$ 距离为 $0.4102(5) \mathrm{nm}$. 有关金属阳离子与聚金属氧酸盐中的 $\mathrm{MoO}_{6}$ 八面体的端氧配位, 在利用水热法合成的 $\left[\left\{\mathrm{Ni}(\text { phen })_{2}\right\}_{2}\left(\xi-\mathrm{Mo}_{8} \mathrm{O}_{26}\right)\right],\left[\left\{\mathrm{Cu}\left(4,4^{\prime}-\text { bipy }\right)\right\}_{4} \mathrm{M}_{\mathrm{O} 8} \mathrm{O}_{26}\right]$ 等化合物中有报道 ${ }^{[5,6]}$. 但是利用金属离子与 Keggin 结构杂多阴离子的端氧配位还是首次报道. 在 Siedle 等合成的 Keggin 结构有机金属阳离子盐 $\left[\left(\mathrm{ph}_{3} \mathrm{P}\right)_{2} \mathrm{IrH}_{2}\right]_{3}\left[\mathrm{PMo}_{12} \mathrm{O}_{40}\right]$ 和 $\left[\left(\mathrm{ph}_{3} \mathrm{P}\right)_{2} \mathrm{Rh}(\mathrm{CO})_{4}\right]_{4}\left[\mathrm{SiW}_{12} \mathrm{O}_{40}\right]$ 中, $\mathrm{Ir} \cdots \mathrm{Mo}$ 和 $\mathrm{Rh} \cdots \mathrm{W}$ 之间的距离大于 $0.4 \mathrm{~nm}$, 因此没有明显的 Ir-O-Mo 和 Rh-O-W 连接 ${ }^{[10,11]}$. 而此前报道的分子基化合物如 $\left[\mathrm{TTF}_{6}\left[\mathrm{XM}_{12} \mathrm{O}_{40}\right]\left(\mathrm{NEt}_{4}\right)(\mathrm{M}=\mathrm{W}\right.$, $\mathrm{Mo} ; \mathrm{X}=\mathrm{P}, \mathrm{Si}$ ) 等 ${ }^{[1]}$, 有机给体与杂多阴离子在结构中处于分隔状态, 没有直接的联系. 此外, 2 个与

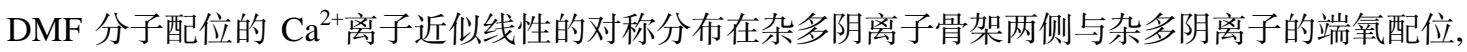
符合分子结构中分子间相互作用力最小的要求. 这使得 2 个毗邻的结构单元利用相邻的金属离子 通过与 DMF 分子配位连接在一起成为可能, 从而为形成一维链状结构创造了条件. 标题化合物所 显示的结构为利用分子设计和分子组装合成新型结构的功能性化合物提供了一种新的思路.

杂多阴离子具有基本 Keggin 结构(见图 3). 标题化合物中 $\mathrm{SiO}_{4}$ 四面体的 4 个 $\mathrm{O}\left(\mathrm{O}_{\mathrm{a}}\right)$ 原子无 序. $\mathrm{SiO}_{4}$ 四面体的每一个氧原子与 6 个 $\mathrm{MoO}_{6}$ 八面体相关, 而每一个 $\mathrm{MoO}_{6}$ 八面体与 $\mathrm{SiO}_{4}$ 四面 


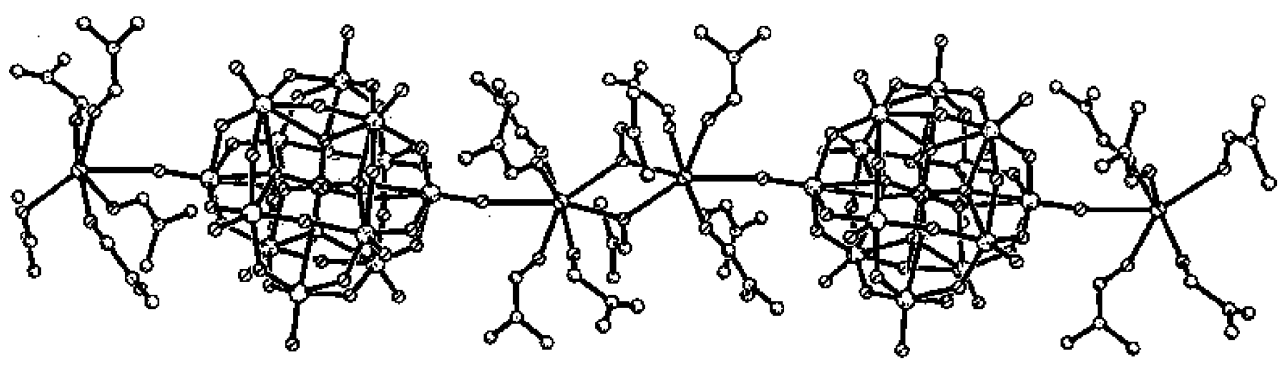

图 2 标题化合物的一维结构图

体中的 2 个氧原子相关. 4 个氧原子位于 2 个平面. $\mathrm{O} 20, \mathrm{O} 20 \mathrm{~A}, \mathrm{O} 22$ 和 $\mathrm{O} 22 \mathrm{~A}$ 在一个平面内, O19, O19A, O21 和 O21A 在一个平面内. O19 和 O19A,O20 和 O20A, O21 和 O21A, O22 和 O22A 之 间的距离分别为 $0.321,0.329,0.335$ 和 $0.321 \mathrm{~nm}$. Si-O 键长在 $0.1603(13), 0.1674(14) \mathrm{nm}$ 之间, 平 均值 $0.1632 \mathrm{~nm}$ 与文献值相当 ${ }^{[12]}$. 在 $\mathrm{MoO}_{6}$ 八面体中, $\mathrm{Mo}-\mathrm{O}$ 键长在较宽范围内变化, 从 0.1637 到 $0.2453 \mathrm{~nm}$. 最短的 Mo-O 键为端基氧键, 键长范围在 0.1637-0.1672 $\mathrm{nm}$ 之间, 平均值 0.1653 $\mathrm{nm}$ 与文献值一致 ${ }^{[12]}$; 最长的 $\mathrm{Mo}-\mathrm{O}$ 键长为 $0.2361 \sim 0.2443 \mathrm{~nm}$, 是连接 $\mathrm{Mo}$ 原子 $\mathrm{Si}$ 原子的桥氧, 平均值 $0.2403 \mathrm{~nm}$ 比文献值长 $0.005 \mathrm{~nm}^{[12]}$; 在连接桥氧的 $\mathrm{Mo}-\mathrm{O}_{\mathrm{b} / \mathrm{c}}$ 键长中, $\mathrm{Mo}-\mathrm{O}_{\mathrm{b} / \mathrm{c}}$ 的键长从 0.1786 到 $0.2011 \mathrm{~nm}$, 平均值 $0.1904 \mathrm{~nm}$ 比文献值短 $0.0022 \mathrm{~nm}^{[12]}$. 以上结构分析结果表明, 杂 多阴离子中 $\mathrm{SiO}_{4}$ 四面体和 $\mathrm{MoO}_{6}$ 八面体都发生了不同程度的畸变, 再一次说明了杂多阴离子 与有机基团之间存在较强的相互作用, 其中, $\mathrm{Mo}-\mathrm{O}_{\mathrm{b} / \mathrm{c}}$ 的键长变化最明显. 以上讨论的不同键 长变化均与标题化合物的红外光谱分析结果相吻合.

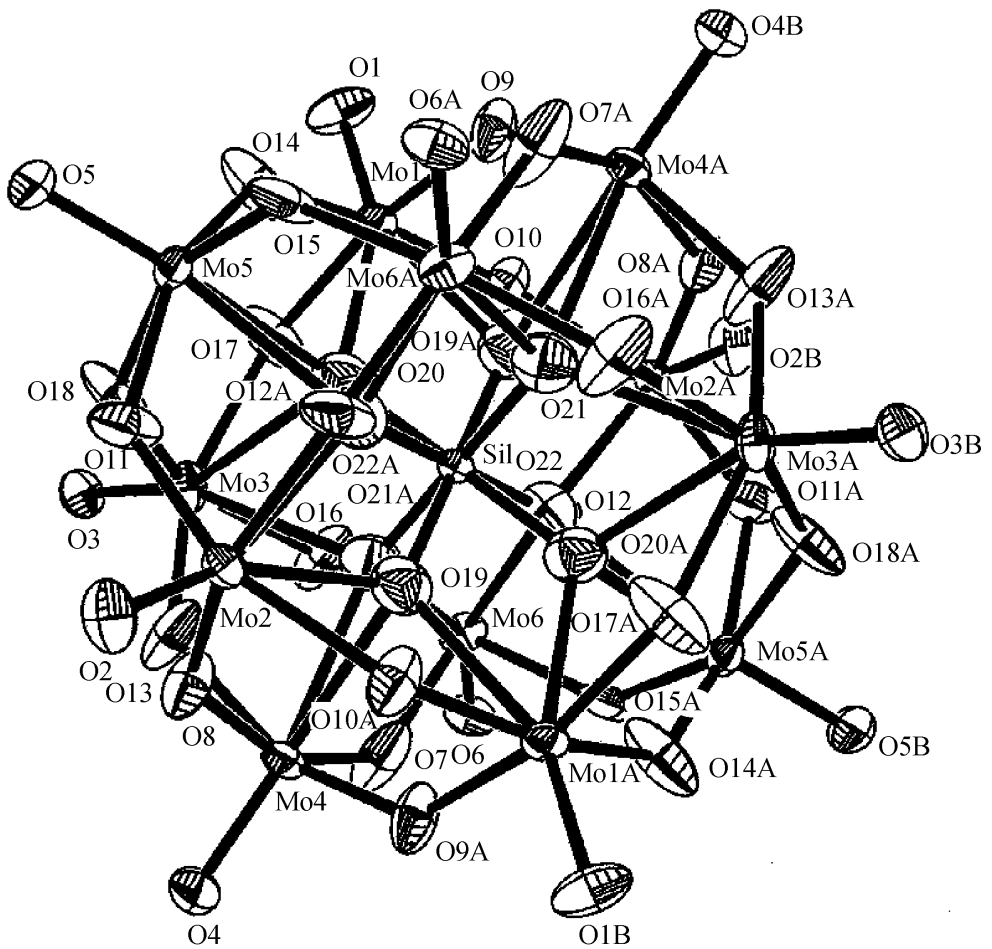

图 3 阴离子结构图 


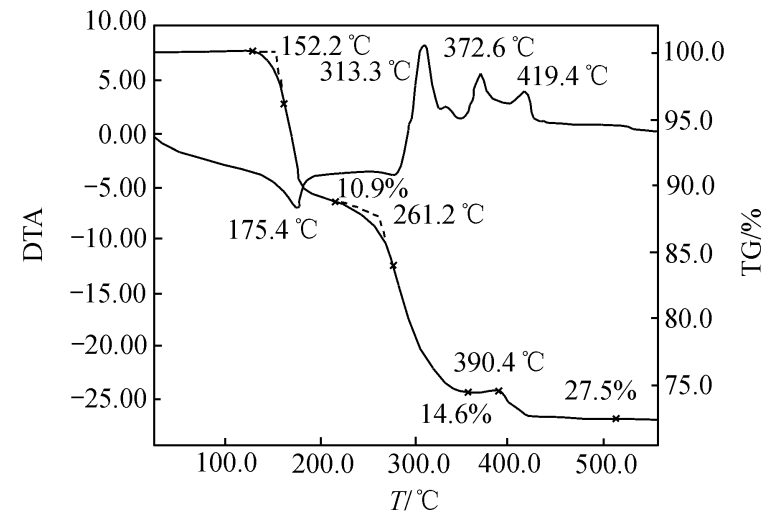

图 4 标题化合物的 TG/DTA 图

\section{5 热性质}

标题化合物的热分解过程从 TG 曲 线可以看出(见图 4), 分三步进行. 第一 步从 $152 \sim 261.2^{\circ} \mathrm{C}$. 化合物失重 $10.9 \%$, 相当于失去 4 分子 $\mathrm{DMF}$ (占总重的 $10.8 \%$ ), 相应的 DTA 曲线在 $175.4^{\circ} \mathrm{C}$ 处 出现一个吸热峰; 第二步失重从 $261.2 \sim 390.4^{\circ} \mathrm{C}$, 失重 $14.6 \%$, 相当于失 去 5.5 分子的 DMF(占总重的 $14.9 \%$ ), 相 应的 DTA 曲线在 $313.3^{\circ} \mathrm{C}$ 和 $372.6^{\circ} \mathrm{C}$ 处出 现 2 个较强的放热峰, 说明此时伴随着

化合物的失重, 化合物中的 DMF 分子开始发生强烈的氧化反应; 第三步失重从 $390.4^{\circ} \mathrm{C}$ 至化 合物恒重, 失重 $2 \%$, 相当于失去剩余的 0.5 分子 DMF(占总重的 $1.4 \%$ ), 相应的 DTA 曲线在 $419.4^{\circ} \mathrm{C}$ 处出现一个较弱的放热峰, 除了表明剩余 DMF 分子的燃烧外, 还表明杂多阴离子骨架 开始分解.

化合物总失重为 $27.5 \%$, 与理论计算总失重值 $27.1 \%$ 基本吻合. 与 $\mathrm{H}_{4} \mathrm{SiMo}_{12} \mathrm{O}_{40} \cdot n \mathrm{H}_{2} \mathrm{O}$ 中 $\left[\mathrm{SiMo}_{12} \mathrm{O}_{40}\right]^{4-}$ 的热稳定性相比 ${ }^{[13]}$, 标题化合物中的杂多阴离子的热稳定性有明显提高. 此外, 值得注意的是, TG 曲线在 350 390 之间有一较弱的增重现象, 可能是连接金属离子与杂多阴 离子端氧的 $\mathrm{Ca}-\mathrm{O}$ 键的断裂后, $\mathrm{Ca}^{2+}$ 离子转化为 $\mathrm{CaO}$ 的缘故.

\section{参考文 献}

1 Coronado E, Gomez-Garcia C J. Polyoxometalate-based molecular materials. Chem Rev, 1998, 98: $273 \sim 296$

2 Gomez-Garcia C J, Gimenez-Saiz C, Triki S, et al. Coexistence of magnetic and delocalized elections in hybrid molecular materials. The series of organic-inorganic radical salts $(\text { BEDT-TTF })_{8}\left[\mathrm{XW}_{12} \mathrm{O}_{40}\right](\operatorname{solv})_{n}$. $\left(\mathrm{X}=2\left(\mathrm{H}^{+}\right), \mathrm{B}^{\mathrm{III}}, \mathrm{Si}^{\mathrm{N}}, \mathrm{Cu}^{\mathrm{II}}, \mathrm{Co}^{\text {II }}, \mathrm{Fe}^{\mathrm{III}}\right.$; solv $=\mathrm{H}_{2} \mathrm{O}, \mathrm{CH}_{3} \mathrm{CN}$ ). Inorg Chem, 995, 34: 4139

3 Coronado E, Galan-Macaros J R, Gimenez-Saiz C, et al. Radical salts of the organic BET-TTF with Polyoxometalate clusters. J Mater Chem,1998, 8(2): $313 \sim 317$

4 Le Magneres P, Ouahab L, Golben S, et al. Diamagnetic and paramagnetic keggin polyoxometalate salts containing 1-D and 3-D decamethylferrocenium networks: preparation, crystal structures, and magnetic properties of $\left[\mathrm{Fe}\left(\mathrm{C}_{5} \mathrm{Me}_{5}\right)_{2}\right]_{4}(\mathrm{POM})(\mathrm{solv})_{n}$ $\left(\mathrm{POM}=\left[\mathrm{SiMo}_{12} \mathrm{O}_{40}\right]^{4-},\left[\mathrm{SiW}_{12} \mathrm{O}_{40}\right]^{4-},\left[\mathrm{PMo}_{12} \mathrm{O}_{40}\right]^{4-},\left[\mathrm{HFeW}_{12} \mathrm{O}_{40}\right]^{4-}\right.$; solv= $\left.\mathrm{H}_{2} \mathrm{O}, \mathrm{C}_{3} \mathrm{H}_{7} \mathrm{ON}, \mathrm{CH}_{3} \mathrm{CN}\right)$. Inorg Chem, 1994, 33: $5180 \sim 5187$

5 徐吉庆, 王仁章, 郭纯孝, 等. 金属-氧族支撑的过渡金属配合物 $\left[\left\{\mathrm{Ni}(\mathrm{phen})_{2}\right\}_{2}\left(\xi-\mathrm{Mo}_{8} \mathrm{O}_{26}\right)\right]$ 的水热合成、结构及量子化学 研究. 中国科学, B 缉, 2001, 31(2): 138 145

6 Hagrman D, Zubieta C, Rose D J, et al. Composite constructed from one-dimensional coordination polymer matrices and molybdenum oxide subunits: Polyoxomolybdate clusters within $\left[\left\{\mathrm{Cu}\left(4,4^{\prime} \text {-bipy }\right)\right\}_{4} \mathrm{M}_{\mathrm{O}_{8}} \mathrm{O}_{26}\right]$ and $\left[\left\{\mathrm{Ni}\left(\mathrm{H}_{2} \mathrm{O}\right)_{2}\left(4,4^{\prime}-\text { bipy }\right)_{2}\right\}_{4} \mathrm{M}_{\mathrm{O} 8^{-}}\right.$ $\left.\mathrm{O}_{26}\right]$ and one-dimensional oxoide chains in $\left[\left\{\mathrm{Cu}\left(4,4^{\prime}\right.\right.\right.$-bipy $\left.\left.)\right\} 4 \mathrm{Mo}_{15} \mathrm{O}_{47}\right] \cdot \mathrm{H}_{2} \mathrm{O}$. Angew Chem Int Ed Engl, 1997, $36: 873 \sim 875$

7 Rocchiccioli-Deltcheer C, Fournier M, Franck R, et al. Vibrational investigations of polyoxometalates. 2. Evidence for anions-anion interactions in molybdenum( vi ) and tungsten( vi) compounds related to the Keggin structure. Inorg Chem, 1983, 22: 207

8 Niu J Y, You X Z, Duan C Y, et al. A novel optical complex between an organic substrate and a polyoxometalate. Crystal and 
molecular structure of $\alpha-\mathrm{H}_{4} \mathrm{SiW}_{12} \mathrm{O}_{40} \cdot 4 \mathrm{HMPA} \cdot 2 \mathrm{H}_{2} \mathrm{O}$ (HMPA=Hexamethylphosphoramide). Inorg Chem, 1996, $35: 4221$

9 Pan L, Frydel T, Sander M B, et al. The effect of pH on the dimensionality of coordination polymers. Inorg Chem, 2001, 40(6): $1271 \sim 1283$

10 Gouzerh P, Proust A. Main-group element, organic, and organometallic derivatives of polymetalates. Chem Rev, 1998, 98: $77 \sim 111$

11 Siedle A R, Gleaso W B, Newmark R A, et al. Solid-state chemistry of molecular metaoxide clusters. Bis(triphenylphosphine) rhordium(I) carbonyl derivatives. Inorg Chem, 1990, 29: $1667 \sim 1673$

12 Wery A S, Gutierrez-Zorrilla J M, Lnque A, et al. Tert-butylammonium silicododecamolybate. Synthesis, vibrational study, crystal structure, thermal behaviour and electrochemical properties. Polyhedron, 1998, 17: 1247

13 王作屏, 牛景扬, 许 林, 等. Keggin 结构杂多酸热性研究. 化学学报, 1995 53(8): $757 \sim 764$ 